\title{
Quantification and Pathogenicity of Candida albicans in Denture-Wearing and Nondenture-Wearing Elderly
}

\author{
${ }^{1}$ Department of Oral Biology and Oral Science Research Center, \\ Faculty of Dentistry, Universitas Indonesia, Jakarta, Indonesia \\ 2Department of Biology, Graduate School, Universitas Nasional, \\ South Jakarta, Indonesia \\ ${ }^{3}$ Department of Biology, Graduate School, Universitas Nasional, \\ South Jakarta, Indonesia
}

Boy M. Bachtiar ${ }^{1}$ Turmidzi Fath ${ }^{2}$ Retno Widowati ${ }^{3}$ Endang W. Bachtiar ${ }^{1}$

\begin{abstract}
Address for correspondence Endang W. Bachtiar, M.Biomed, Ph.D, Department of Oral Biology and Oral Science Research Center, Faculty of Dentistry, Universitas Indonesia, JL. Salemba Raya 4, Jakarta 10430, Indonesia (e-mail: endang04@ui.ac.id).
\end{abstract}

Eur J Dent:2020;14:423-428

\begin{abstract}
Keywords

- Candida albicans

- tongue

- denture

$-A L S 3$

$-H W P 1$

$-Y W P 1$
\end{abstract}

Objective The primary purpose of this study was to evaluate and compare the microbial loads and pathogenicity traits of oral Candida albicans in denture-wearing (DW; $n=15$ ) and nondenture-wearing (NDW; $n=15$ ) elderly persons.

Materials and Methods The fungal counts of the saliva, tongue dorsa, and prosthesis-fitting surfaces of the participants were assessed using real-time polymerase chain reaction to compare the quantity and expression of selected C. albicans biofilmassociated genes (ALS3, HWP1, and YWP1).

Statistical Analysis The obtained data were analyzed by one-way analysis of variance, followed by Bartlett's test. When appropriate, the Student's t-test was also used; a value of $p<0.05$ was considered statistically significant.

Results In both groups, the count of $C$. albicans was found to be significantly higher in saliva than in other oral samples. The expression of the hypha-specific genes (ALS3 and HWP1) in the tongue dorsa was higher in the DW group $(p<0.05)$, whereas the transcription level of the yeast-specific gene (YWP1) was significantly higher in the NDW group.

Conclusion Both tongue dorsa and dentures appear to be sharing factors that are important for $C$. albicans biofilm growth in abiotic and biotic oral surfaces of the elderly.

\section{Introduction}

Candida albicans is a unique opportunistic fungal species that, together with other oral microflora, can exist in biofilms, on either host tissues or abiotic surfaces in humans. ${ }^{1,2}$ Since biofilm formation is a fungal virulence trait, C. albicans colonization and biofilm formation on oral mucosa, tooth surfaces, and dental prostheses are of clinical significance. From a prosthetic oral rehabilitation perspective, denture-wearing (DW) should be considered a predisposing factor for oral candidiasis. ${ }^{3}$ Therefore, knowledge of pathologies related to prosthetic microbial plaque is essential for maintaining oral health, particularly because oral physiological changes such as impaired salivary flow commonly occur in the elderly. Subsequent to these changes, $C$. albicans grows in hypha form due to the lack of a salivary component called statherin, which inhibits hyphal growth by inducing the transition of hyphae to lateral yeast cells. ${ }^{4}$ In this context, following our previous study on detecting C. albicans in children with early childhood caries experience, ${ }^{5}$ we aimed to further investigate the characteristics of $C$. albicans pathogenicity in elderly subjects. Therefore, we determined the extent to which C. albicans pathogenicity is affected by denture insertion in elderly people. We used real-time polymerase chain reaction (PCR) to assess the fungal load and the expression of selected biofilm-associated genes (ALS3, HWP1, and YWP1). We analyzed whether the genes were transcribed at similar levels in different oral niches (tongue dorsa and prosthesis-fitting surfaces) of DW and nondenture-wearing (NDW) elders.

License terms

()(1) $\ominus \circledast$ 


\section{Materials and Methods}

\section{Participants}

Thirty elderly adults, aged $\geq 60$ years (male and female), were randomly selected for this study. They were living in two geriatric homes located in a rural area of Jakarta, the capital city of Indonesia. Participants who had illnesses or medical problems (systemic diseases, pharmacological therapies, etc.) were not included in the study.

Samples of their saliva, dentures, and tongue dorsa were obtained ( - Fig. 1). The participants were categorized into two groups: denture wearers $(n=15)$, with maxillary or mandibular dentures (partial or full), and nondenture wearers $(n=15),{ }^{6,7}$

Other inclusion criteria were no smoking, no fasting, and no use of antibiotics for 3 months before this study. The participants were instructed not to eat and/or drink for at least 2 hours before the oral sample collection.

The samples were collected, after informed consent was obtained from all the participants, in accordance with the approved protocol of the Bioethics Committee of the Faculty of Dentistry, Universitas Indonesia (protocol number 020950818). The protocol conformed to the criteria of the Helsinki Declaration and the good clinical practical guidelines of the International Council on Harmonization.

Unstimulated saliva (minimum volume of $2 \mathrm{~mL}$ ) was collected by spitting into a sterile Falcon tube. The other oral samples (from dentures and tongue dorsal surfaces) were collected using a sterile cotton swab. Samples from dentures were collected by swabbing the mucosal surface five times; for those from the tongue dorsa, the swabbing was done vertically (five times), from the circumvallate papillae to the tip of the tongue. All the collected samples were then placed into an Eppendorf tube and immediately cold-transported to the laboratory, where the samples were stored at $-80^{\circ} \mathrm{C}$ until further use. For our analysis, we focused on the quantity of C. albicans and the mRNA expression of selected biofilmassociated genes (ALS3, HWP1, and YWP1) in the tongue surfaces, denture-fitting surfaces, and saliva. We also analyzed the association between the $C$. albicans count and the transcription level of each gene tested.

\section{Quantification of C. albicans by qPCR}

The method used to extract fungal genomic DNA from each sample was the same as that reported previously using the Trizol reagent (Sigma-Aldrich; Dorset, UK). ${ }^{5}$ The concentration and quality of the obtained DNA were determined using Qubit assay reagents (Invitrogen; Carlsbad, California, United States); after dissolving in Tris-EDTA buffer, the DNA was cooled to $-20^{\circ} \mathrm{C}$ for further analysis. Then, the DNA samples were quantified through quantitative PCR (qPCR) with the C. albicans-specific primers ${ }^{22}$ shown in - Table 1. For PCR quantification, each sample was run in triplicate on an ABI StepOnePlus Real-Time PCR System, wherein an SYBR Green PCR Master Mix (Applied Biosystems) was used in accordance with the protocol provided by the company.

The PCR conditions were a final reaction volume of $10 \mu \mathrm{L}$ (composed of $50 \mathrm{ng}$ of sample DNA and $1 \mu \mathrm{M}$ of primer) and thermal cycling conditions of a 10-minute initial denaturation at $95^{\circ} \mathrm{C}$, followed by 40 cycles of denaturation at $95^{\circ} \mathrm{C}$ for 15 -second annealing at $60^{\circ} \mathrm{C}$ for 60 second and elongation at $95^{\circ} \mathrm{C}$ for 15 seconds. The qPCR product was visualized as a melting curve $\left(95^{\circ} \mathrm{C}\right.$ for 15 seconds, $60^{\circ} \mathrm{C}$ for 60 seconds, and $95^{\circ} \mathrm{C}$ for 15 seconds) and the cycle threshold $\left(C_{t}\right)$ value was converted to log colony forming unit (CFU).

The amount of genomic DNA of $C$. albicans was estimated by constructing a standard curve with $r^{2}$, as shown in - Fig. 2A. Briefly, we performed a 10-fold serial dilution of extracted $C$. albicans (ATCC 10231). The number of viable fungal cells (CFU/mL) was assessed by plating culture dilutions on Sabouraud dextrose agar, and the same strain was used as a positive control while running the qPCR. Therefore, the amount of $C$. albicans in the oral samples was determined by plotting the $C_{t}$ values against the log of the standard curve, whereas the melting peaks were used to assess the specificity of the amplicon ( - Fig. 2B-D).
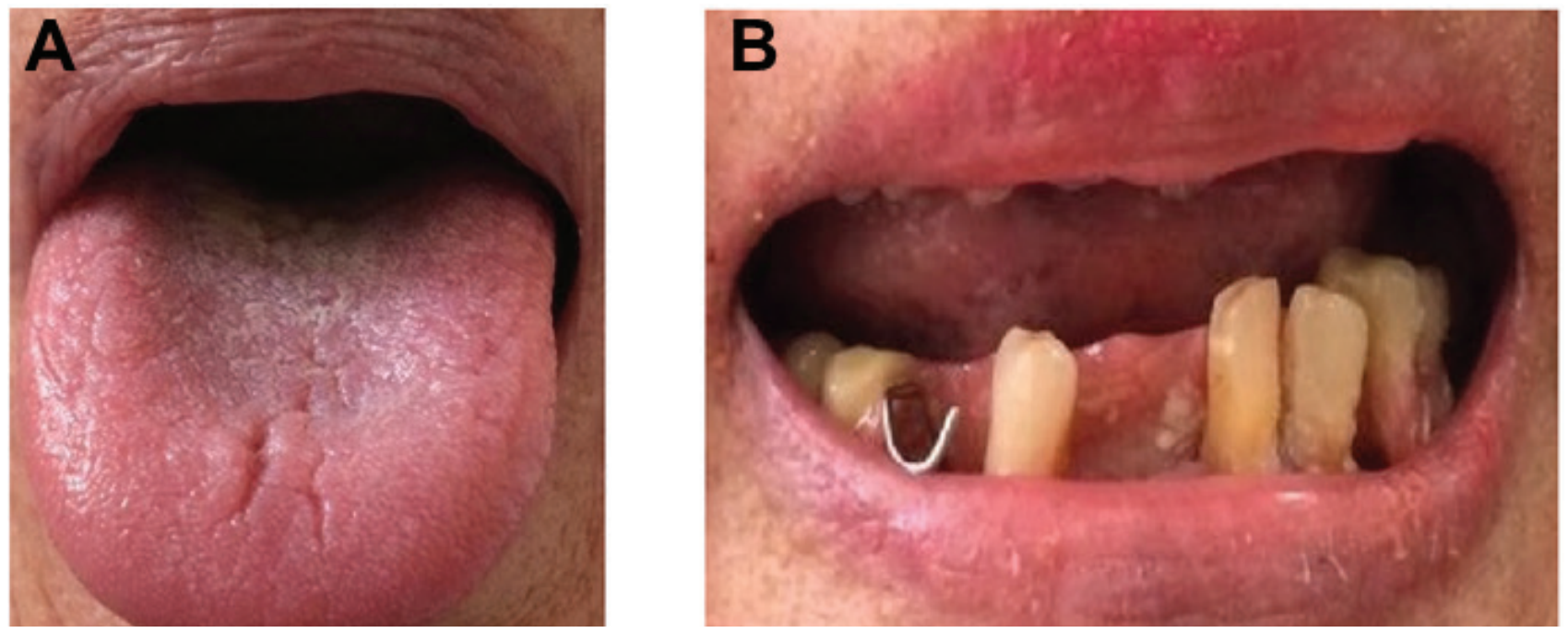

Fig. 1 Sample profiles of elderly subjects. Tongue dorsa (A) and dentures (B). 
Table 1 Primers used in this study

\begin{tabular}{|c|c|c|}
\hline Primer & Sequences & Reference \\
\hline \multirow[t]{2}{*}{ ACT1 } & F: 5'-TTTCATCTTCTGTATCAGAGGAACTTATTT-3' & \multirow[t]{2}{*}{8} \\
\hline & R: 5'-ATGGGATGAATCATCAAACAAGAG -3' & \\
\hline \multirow[t]{2}{*}{$18 \mathrm{~S}$ rRNA } & F: 5'- CACGACGGAGTTTCACAA GA-3' & 22 \\
\hline & R: 5' - CGA TGG AAG TTT GAG GCA AT-3' & \\
\hline \multirow[t]{2}{*}{ YWP1 } & F: 5' - GCTACTGCTACTGGTGCTA-3' & \multirow[t]{2}{*}{22} \\
\hline & R: 5'- AACGGTGGTTTCTTGAC-3' & \\
\hline \multirow[t]{2}{*}{ HWP1 } & F: 5'- GCTCCTGCTCCTGAAATGAC-3' & \multirow[t]{2}{*}{22} \\
\hline & R: 5' - CTGGAGCAATTGGTGAGGTT-3' & \\
\hline \multirow[t]{2}{*}{ ALS3 } & F: 5' - CAACTTGGGTTATTGAAACAAAAACA-3' & \multirow[t]{2}{*}{22} \\
\hline & R: 5' - AGAAACAGAAACCCAAGAACAACC-3' & \\
\hline
\end{tabular}
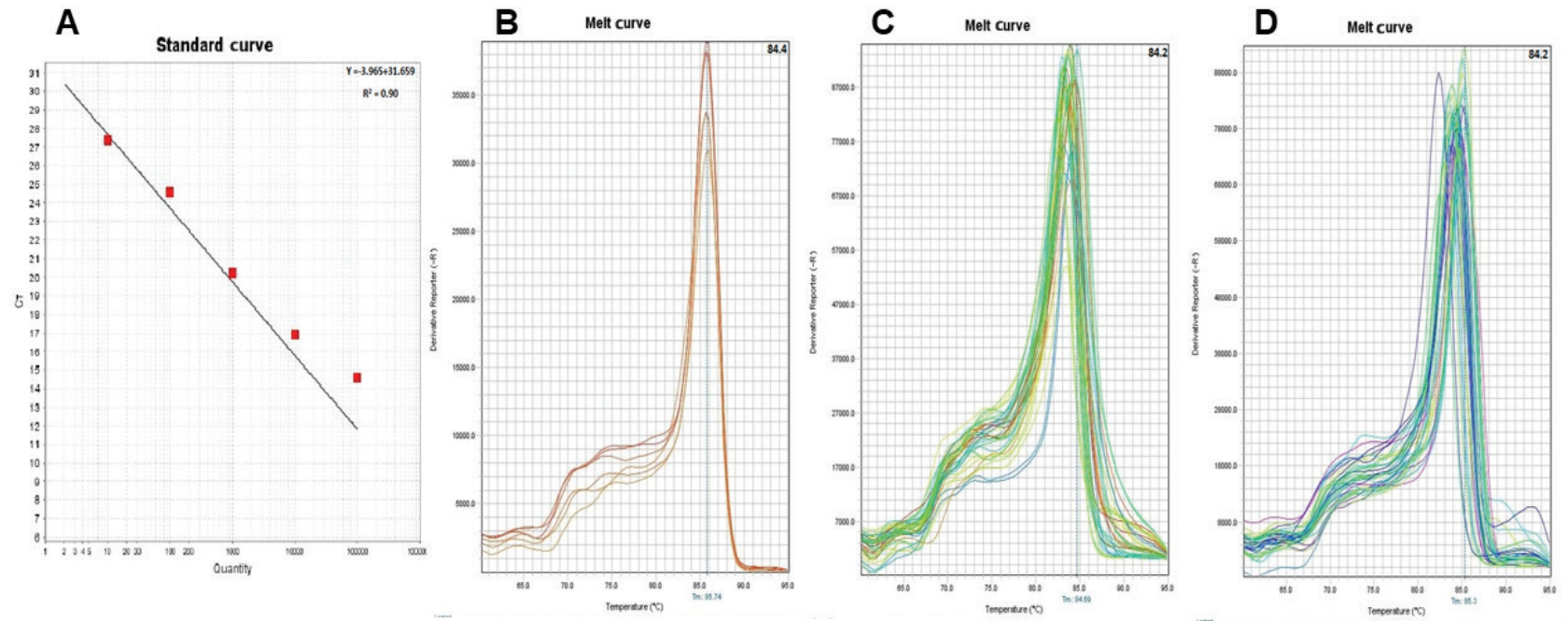

Fig. 2 Standard curves and melt curves. Standard curve of Candida albicans ATCC 10231 (A) and representative melt curve graphs for C. albicans count in saliva (B), tongue dorsum (C), and denture mucosal surface (D).

\section{qPCR Analysis for mRNA Expression of C. albicans ALS3, HWP1, and YWP1 in Tongue Dorsa and Denture Samples}

RNA isolation, purification, and reverse transcription of cDNA were performed similar to the method used in a previous study. ${ }^{5}$ Platinum SYBR Green qPCR SuperMix-UDG (Invitrogen Life Technologies, Carlsbad, California, United States), a passive reference (ROX, Invitrogen), and primers for each gene tested (-Table 1), as well as $1 \mu \mathrm{g}$ of cDNA, were used to quantify the cDNA. The qPCR cycling conditions consisted of a 10 -minute initial denaturation at $95^{\circ} \mathrm{C}$ followed by 40 PCR cycles of 15 seconds at $95^{\circ} \mathrm{C}$ and 1 minute at $60^{\circ} \mathrm{C}$. The formula of fold change $2^{-\Delta \Delta C t}$ was used to calculate the relative mRNA expression, which was compared with that of the housekeeping gene, $A C T 1^{8}$; the mRNAs of genes (ALS3, HWP1, and YWP1) expressed in the saliva samples were used as controls.

\section{Statistical Analysis}

All means are reported as mean \pm standard error. Statistical comparison between the three oral samples tested was done using ordinary one-way analysis of variance, followed by the Bartlett's test. The Mann-Whitney U test was used to compare the saliva and tongue dorsa samples of the two groups. Differences were considered significant if $p$-value was less than 0.05. The data were analyzed using Prism version 6.0 (GraphPad Software, Inc.; San Diego, California, United States).

\section{Result}

Candida albicans was present in all the samples collected from both the groups of participants. In general, although interindividual variation was observed in both groups, the count of $C$. albicans in saliva samples was higher than that in other oral samples ( - Fig. $\mathbf{3 A}$ and $3 \mathrm{~B})(p<0.05)$. Moreover, the amount (log DNA copies) of $C$. albicans in the saliva of the DW group was significantly higher than that of the NDW group ( $p<0.05)$. Lastly, although the tongue dorsa samples of subjects in the NDW group showed, on average, a slightly higher count of $C$. albicans than that of the DW group, this difference was not statistically significant. 
A

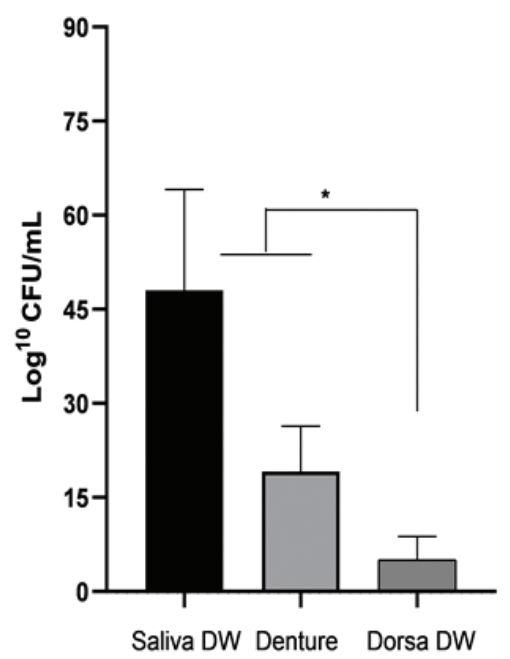

B

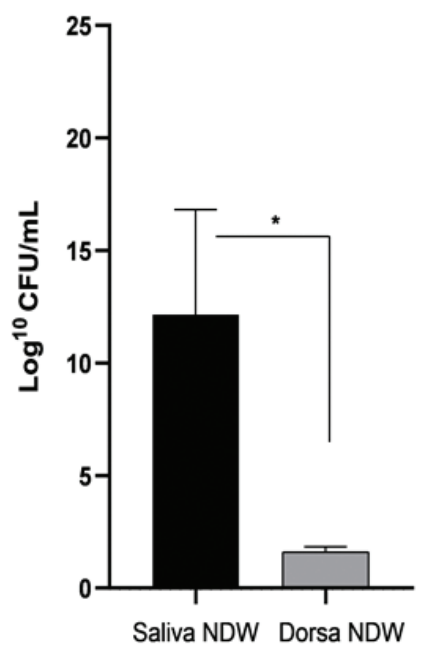

Fig. 3 Standard error of absolute numbers of Candida albicans detected in oral samples (saliva, dentures, and tongue dorsa) of denturewearing (A) and nondenture-wearing (B) elderly subjects. ${ }^{*} p<0.05$. DW, denture wearing; NDW, nondenture wearing.

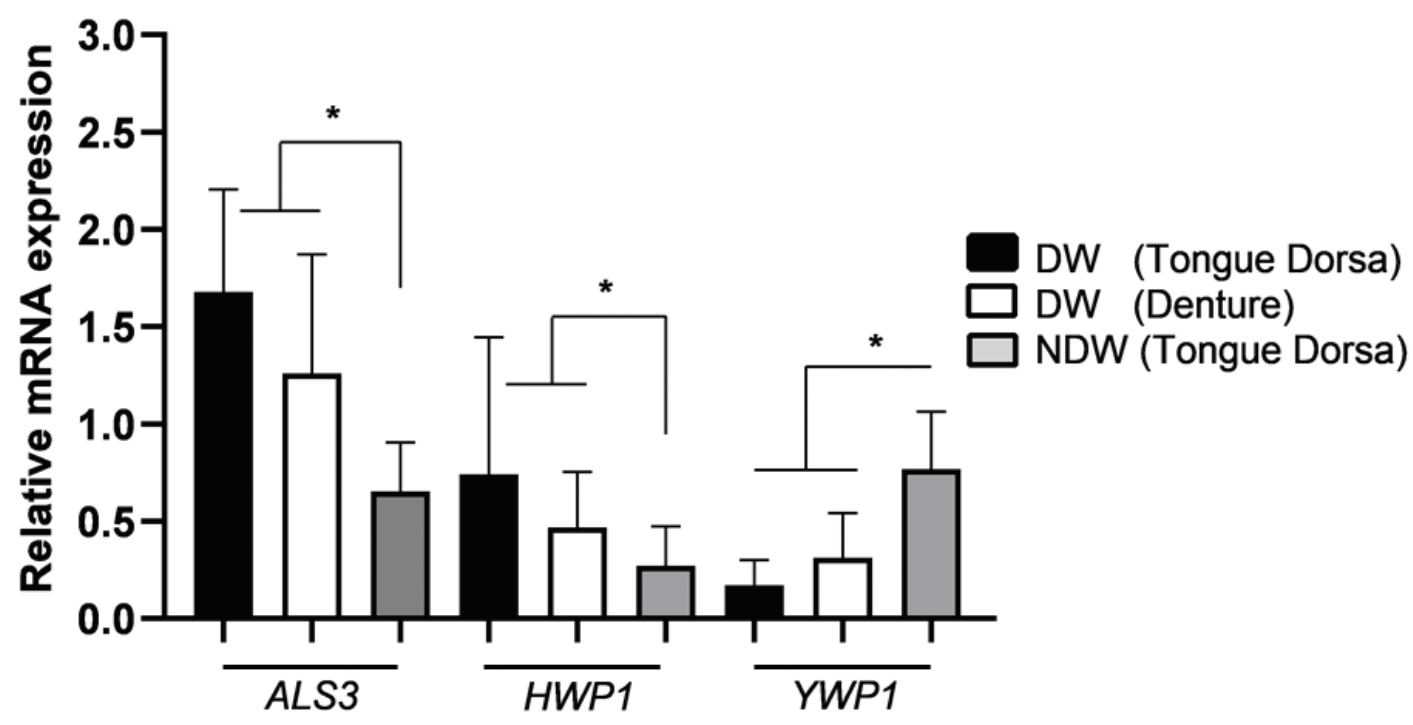

Fig. 4 mRNA expression of selected biofilm-associated genes (ALS3, HWP1, and YWP1). Fold changes in gene expression on tongued dorsa of denture-wearing (A) and nondenture-wearing (B) elders were compared. All quantitative polymerase chain reaction values were normalized according to the expression of the housekeeping gene ACT1. Error bars show standard error from triplicate determinations. ${ }^{*} p<0.05$. DW, denture-wearing; NDW, nondenture-wearing.

\section{Transcription Levels of C. albicans ALS3, HWP1, and YWP1}

We further analyzed the mRNA expression of selected C. albicans genes (ALS3, HWP1, and YWP1, which are involved in the regulation of the fungus phenotypic transition ${ }^{9}$ ) in the tongue dorsa of the DW and NDW subjects. The transcription levels of both ALS3 and HWP1 were significantly higher in the tongue dorsa of the DW subjects than in the NDW subjects. In contrast, a significantly higher level of YWP1 mRNA expression was detected in the tongue dorsa of the NDW subjects compared with that in the DW subjects ( - Fig. 4).

\section{Discussion}

The specific aim of this study was to compare the load and pathogenic traits of oral C. albicans in DW and NDW elderly persons. In both groups, saliva is the oral niche that has the highest count of $C$. albicans. Hence, although saliva plays a key role in modulating fungal colonization in the oral cavity, ${ }^{10}$ the results of this study show that the overall activity of salivary secretion toward C. albicans is less effective in older people. This may indicate age-induced changes in the normal protective functions of saliva (cleansing, lubrication, and antibacterial activity). 
A study by Leung et a ${ }^{11}$ showed that low salivary flow rate (SFR) in the elderly contributes significantly to C. albicans colonization in the oral cavity. Therefore, denture wearers are more at risk of contracting oral candidiasis. ${ }^{12}$ In this study, we did not measure the SFR. However, the high count of salivary C. albicans we observed does not indicate that a low SFR has a significant effect on salivary colonization by this fungus, a phenomenon that has been reported previously. ${ }^{13}$ Hence, it is possible that our elderly subjects may have produced saliva containing complementary receptors for $C$. albicans attachment on the inner surface of the dentures. This attachment may involve salivary pellicles (e.g., proline-rich proteins) that act as complementary receptors to which their ligands, known as cell surface molecule adhesins (e.g., mannoproteins and fibrillar adhesins), bind. ${ }^{14,15}$

Since saliva has been the preferred oral sample in epidemiological studies, ${ }^{16}$ we analyzed the difference in the amount of C. albicans in saliva collected from the DW and NDW groups. First, we found that there was a significantly higher C. albicans load in the saliva samples from both the DW and NDW groups, compared with that in the other oral samples. We also observed that the C. albicans count was highest in the saliva of the DW group. We hypothesize that the presence of dentures in DW subjects provides environmental conditions, particularly beneath the denture base, that may favor yeast colonization in the oral cavity. Therefore, both the tongue dorsa and the fitting surfaces of dental prostheses must be considered as important reservoirs of $C$. albicans colonization, from which this microorganism dislodges into saliva. Moreover, the results of this study showed that the C. albicans counts on the tongue surfaces of the DW and NDW groups are similar. This may indicate that the oral conditions resulting from DW do not affect the colonization status of the fungus on biotic surfaces (tongue dorsa). Interestingly, within the DW group, C. albicans shows a lower affinity for adherence to the tongue dorsa surfaces than to the mucosa-fitting surfaces of the dentures. This result and those of other studies ${ }^{17,18}$ suggest that in elderly subjects, a nonshedding abiotic substratum is an ideal oral habitat for microbial attachment and growth.

Since oral candidiasis occurs on the oral mucosal surface, ${ }^{19}$ we further compared the pathogenicity of C. albicans in tongue dorsa by analyzing the expression of selected biofilm-associated genes in DW and NDW persons. We chose the ALS3, HWP1, and YWP1 genes because the $C$. albicans load in tongue dorsa is associated with an alteration in fungal morphology (from yeast to hyphal form), and upregulation or downregulation of the hypha-specific gene (HWP1), adhesion-related gene $(A L S 3),{ }^{20}$ and gene involved in the antiadhesive activity of the fungus (yeast-associated gene, YWP1)..1

The transcription levels of ALS3 and HWP1 were higher in the DW group than those in the NDW group. Interestingly, YWP1 was higher expressed in the tongue dorsa of the NDW group than in the DW group. Although a similar overall carriage rate of $C$. albicans was found in the tongue dorsa of both the groups, the difference in the expression of the selected biofilm-associated genes did not affect the colonization status of the fungus. The dissimilarities between the DW and NDW groups in the expression of both hypha and yeast-associated genes suggested that the NDW subjects harbored stable yeast cells as biofilm components on their tongue dorsa surfaces. Since yeast cells play an important role in the commensalisms and pathogenesis of C. albicans, ${ }^{21}$ the tongue was the primary reservoir for $C$. albicans for the NDW subjects that participated in this study.

\section{Conclusions}

This preliminary study revealed the difference in the pathogenicity of C. albicans (evaluated by qPCR) in DW and NDW elderly persons. The results showed that whole saliva is a better oral sample for detecting C. albicans in elderly persons, irrespective of wearing dental prostheses. Furthermore, the adhesion of $C$. albicans to the biotic surfaces of oral cavities is affected by the capacity of the fungus to colonize the inner surfaces of dentures, which ultimately improves its ability to form biofilms on tongue dorsa and become pathogenic in oral environments. These preliminary data, therefore, provide a justification and basis for further exploration of other oral fungal colonization phenomena, particularly, in broader groups of elderly subjects.

\section{Funding}

This work was supported by a research grant for international publication (Q1Q2) provided by Universitas Indonesia with the number NKB-0244/UN2.R3.1/HKP. 05.00/2019.

\section{Conflict of Interest}

None declared.

\section{Acknowledgments}

The authors would like to thank Vivi, Asti, and Anissa for their help in the laboratory work and Ifadah for his contribution in collecting the samples. We would also like to thank Editage for English language editing.

\section{References}

1 Nett J, Andes D. Candida albicans biofilm development, modeling a host-pathogen interaction. Curr Opin Microbiol 2006;9(4):340-345

2 Mathé L, Van Dijck P. Recent insights into Candida albicans biofilm resistance mechanisms. Curr Genet 2013;59(4):251-264

3 Akpan A, Morgan R. Oral candidiasis. Postgrad Med J 2002;78( 922):455-459

4 Leito JT, Ligtenberg AJ, Nazmi K, Veerman EC. Identification of salivary components that induce transition of hyphae to yeast in Candida albicans. FEMS Yeast Res 2009;9(7):1102-1110

5 Bachtiar EW, Bachtiar BM. Relationship between Candida albicans and Streptococcus mutansin early childhood caries, evaluated by quantitative PCR. F1000 Res 2018;7:1645 
6 Ramage G, Tomsett K, Wickes BL, López-Ribot JL, Redding SW. Denture stomatitis: a role for Candida biofilms. Oral Surg Oral Med Oral Pathol Oral Radiol Endod 2004;98(1):53-59

7 Altarawneh S, Bencharit S, Mendoza L, et al. Clinical and histological findings of denture stomatitis as related to intraoral colonization patterns of Candida albicans, salivary flow, and dry mouth. J Prosthodont 2013;22(1):13-22

8 Alonso GC, Pavarina AC, Sousa TV, Klein MI. A quest to find good primers for gene expression analysis of Candida albicans from clinical samples. J Microbiol Methods 2018;147:1-13

9 Huang G. Regulation of phenotypic transitions in the fungal pathogen Candida albicans. Virulence 2012;3(3):251-261

10 Hibino K, Samaranayake LP, Hägg U, Wong RW, Lee W. The role of salivary factors in persistent oral carriage of Candida in humans. Arch Oral Biol 2009;54(7):678-683

11 Leung KP, Crowe TD, Abercrombie JJ, et al. Control of oral biofilm formation by an antimicrobial decapeptide. J Dent Res 2005;84(12):1172-1177

12 Wiener RC, Wu B, Crout R, et al. Hyposalivation and xerostomia in dentate older adults. J Am Dent Assoc 2010;141(3):279-284

13 Nadig SD, Ashwathappa DT, Manjunath M, Krishna S, Annaji AG, Shivaprakash PK. A relationship between salivary flow rates and Candida counts in patients with xerostomia. J Oral Maxillofac Pathol 2017;21(2):316

14 Radford DR, Sweet SP, Challacombe SJ, Walter JD. Adherence of Candida albicans to denture-base materials with different surface finishes. J Dent 1998;26(7):577-583
15 Cavalcanti YW, Wilson M, Lewis M, et al. Salivary pellicles equalise surfaces' charges and modulate the virulence of Candida albicans biofilm. Arch Oral Biol 2016;66:129-140

16 Nasidze I, Li J, Quinque D, Tang K, Stoneking M. Global diversity in the human salivary microbiome. Genome Res 2009;19(4):636-643

17 Cavalheiro M, Teixeira MC. Candidabiofilms: threats, challenges, and promising strategies. Front Med (Lausanne) 2018;5:28

18 Gacon I, Loster JE, Wieczorek A. Relationship between oral hygiene and fungal growth in patients: users of an acrylic denture without signs of inflammatory process. Clin Interv Aging 2019;14:1297-1302

19 Basson NJ, van Wyk CW. The establishment of a community of oral bacteria that controls the growth of Candida albicans in a chemostat. Oral Microbiol Immunol 1996;11(3):199-202

20 Hoyer LL, Green CB, Oh SH, Zhao X. Discovering the secrets of the Candida albicans agglutinin-like sequence (ALS) gene family-a sticky pursuit. Med Mycol 2008;46(1):1-15

21 Granger BL. Insight into the antiadhesive effect of yeast wall protein 1 of Candida albicans. Eukaryot Cell 2012;11(6):795-805

22 Feldman M, Ginsburg I, Al-Quntar A, Steinberg D. Thiazolidinedione-8 alters symbiotic relationship in C. albicans-S. mutans dual species biofilm. Front Microbiol 2016;7:140 